\title{
El tratamiento de los elementos lexicalizados en la lexicografía española monolingüe ${ }^{1}$
}

\author{
María Bargalló Escrivá \\ José Caramés Díaz \\ Verónica Ferrando Aramo \\ José A. Moreno Villanueva \\ Universitat Rovira i Virgili
}

\section{INTRODUCCIÓN}

El avance de la técnica lexicográfica en estos últimos años resulta un hecho incuestionable. La voluntad de ofrecer a través de los diccionarios una imagen lo más detallada posible de la lengua ha supuesto un mayor rigor en la información que se ofrece, fruto de una reflexión teórica previa. Al mismo tiempo, se ha prestado una atención considerable a todos aquellos aspectos que pueden facilitar el manejo de este tipo de obras.

A todo ello ha contribuido, sin duda, la mayor permeabilidad que muestra la lexicografía respecto a nuevas propuestas teóricas relacionadas con el estudio del léxico, sin que ello suponga -como es evidenteuna aplicación directa, sin filtros, de estos planteamientos.

Uno de los aspectos que permite mostrar este proceso es el tratamiento de los elementos lexicalizados. Según señalan autores como Martínez Marín (1991, 1996) y Corpas (1997), el estudio teórico de los elementos lexicalizados o, si se quiere, de la fraseología, ha tenido un desarrollo considerable a partir de la década de los 70, pero las dificultades observadas en la clasificación de estos elementos han impedido su aplicación inmediata a la lexicografía. Tal como comenta el profesor

${ }^{1}$ Este trabajo ha sido posible gracias a una subvención de la DGICYT al Proyecto de Investigación El tratamiento de los elementos lexicalizados en un diccionario bilingüe (PS95-0153). Una primera versión de este estudio fue presentado en el XXVII Simposio de la Sociedad Española de Lingüística celebrado en Palma de Mallorca (diciembre, 1997). 
Alvar Ezquerra en la introducción de Corpas (1997, p. II):

Cuando se está produciendo una profunda transformación en la lexicografía, tanto en la teórica como en la práctica, los diccionaristas echan de menos que no se hayan delimitado y definido con claridad los diversos tipos de unidades fraseológicas, para saber cuáles habrían de aparecer en sus obras y cuáles han de ser el objeto de otra clase de repertorios; y las que se han ido incluyendo no responden a un programa metódico y coherente, sino a la buena intención y al saber hacer de los redactores de los diccionarios.

Estas observaciones concuerdan con el resultado del análisis de elementos fraseológicos que realizó Martínez Marín (1991) en una serie de diccionarios; aunque apunta síntomas de modernidad en algunos de ellos, faltan -en su opinión- los elementos teóricos de apoyo para poder alcanzar el rigor que exige la ciencia lingüística actual.

Con el fin de observar los cambios que se han producido desde entonces en el tratamiento de los elementos lexicalizados, nos centraremos en el estudio de los diccionarios monolingües; concretamente, en los diccionarios generales y en los didácticos ${ }^{2}$.

El corpus de expresiones que tomamos como referencia comprende dos tipos de unidades fraseológicas que están despertando el interés de los investigadores en lexicología, así como en lexicografía: las colocaciones y las locuciones. En el estudio de Corpas (1997) estas unidades conforman un grupo en el que aparecen reunidas aquellas unidades "[...] que necesitan combinarse con otros signos lingüísticos y que equivalen a sintagmas" (p. 51). Entiende por colocaciones aquellas

[...] unidades fraseológicas que, desde el punto de vista del sistema de la lengua, son sintagmas completamente libres, generados a partir de reglas, pero que, al mismo tiempo, presentan cierto grado de restricción combinatoria determinada por el uso (cierta fijación interna). Este rasgo esencial distingue las colocaciones de las combinaciones libres de palabras (Corpas, 1997, p. 53).

${ }^{2}$ Quedan pendientes de análisis otros dos tipos fundamentales de obras lexicográficas en relación con este tema: aquellas que se dedican de manera específica a la fraseología (como Varela y Kubarth 1996) y los diccionarios bilingües. Sobre estos últimos, cf. Bargalló, Caramés, Ferrando y Moreno (en prensa), donde se analiza el tratamiento de las unidades fraseológicas en este tipo de diccionarios. 
En el conjunto de las locuciones, por otra parte se incluyen aquellas

[...] unidades fraseológicas del sistema de la lengua con los siguientes rasgos distintivos: fijación interna, unidad de significado y fijación externa pasemática. (Ibid., p. 88) ${ }^{3}$.

\section{PRESENTACIÓN DE LOS DICCIONARIOS}

Para el estudio lexicográfico concreto hemos seleccionado un total de seis diccionarios aparecidos en esta última década. La elección de estas obras responde al hecho de que algunos de los repertorios lexicográficos más significativos anteriores a los 90 ya han sido objeto de estudio ${ }^{4}$. Además, el avance vivido por la lexicografía en estos últimos años -tal como señalamos al principio- justifica que pueda incidirse de nuevo en el problema de la inclusión de la fraseología en el diccionario.

Los repertorios han sido escogidos teniendo en cuenta su tipología. En este sentido, hemos seleccionado dos diccionarios generales ${ }^{5}$ y cuatro diccionarios didácticos de nivel superior 6 , no restringidos únicamente a los niveles escolares. La elección de este último tipo viene condicionada por la especial atención que éstos prestan a la selección del corpus, lo cual puede incidir en el tratamiento de las unidades fraseológicas; de ahí su mayor número en nuestro estudio.

\section{TRATAMIENTO LEXICOGRÁFICO DE LA FRASEOLOGÍA}

En el desarrollo de nuestro estudio analizaremos, en primer lugar, la información contenida en las páginas preliminares y apéndices complementarios que figuran en los diccionarios seleccionados. Consideraremos después de qué manera se incluyen en ellos las expresiones que hemos

${ }^{3}$ Debe señalarse que este último concepto de locución es más amplio que el tradicional, puesto que incluye también los denominados modismos y frases hechas.

${ }^{4}$ Cf. Martínez Marín (1991).

5 Utilizamos el Diccionario de la lengua española (1992) de la Real Academia (DrAE en adelante) y el Gran Diccionario de la Lengua Española (1996, LAROUSSE en adelante).

6 Son, concretamente, el Diccionario Esencial Santillana de la Lengua Española (1991, EsENCIAL en adelante), el Diccionario para la Enseñanza de la Lengua Española (1995, DiPElE), el Diccionario Salamanca de Lengua Española (1996, SAlAMANCA) y el Clave. Diccionario de Uso de Español Actual (1996, ClAVE). 
tomado como punto de referencia. Pretendemos observar, de este modo, cómo se refleja el interés por los elementos lexicalizados en la práctica lexicográfica interna.

\subsection{INFORMACIÓN CONTENIDA EN LAS PÁGINAS PRELIMINARES Y APÉNDICES}

Los diferentes diccionarios estudiados aluden en sus primeras páginas y apéndices complementarios a la inclusión de la fraseología; sin embargo, la consideración que reciben las unidades que se inscriben bajo ese marbete, su clasificación y definición -cuando existen- distan de ser uniformes.

El desigual interés se manifiesta, en primer lugar, en el espacio destinado a las indicaciones sobre este particular, aunque éste no es siempre un elemento decisivo: en el ESENCIAL y en el DRAE apenas se les destina un par de párrafos, en absoluto clarificadores; en el DIPELE y en el LAROUSSE no se les dedica mucho más, pero se introducen oportunas puntualizaciones sobre la clasificación y lematización de las distintas unidades; por último, sólo en el ClaVE y en el SALAMANCA se incluye un apartado específico destinado a la fraseología, siendo especialmente relevante la incorporación -en el primero de estos repertorios- de un apéndice en el que se ordenan alfabéticamente las cerca de 7.000 locuciones incluidas y se señala el artículo en el que están definidas y ejemplificadas.

3.1.1. Clasificación y definición de las unidades fraseológicas.-

El elemento que mejor ilustra la falta de uniformidad en el tratamiento de la fraseología es la diversidad terminológica que se puede observar en los diccionarios para referirse a las unidades que forman parte de ella. No es nuestro objetivo en este trabajo analizar la validez conceptual de las denominaciones y clasificaciones ofrecidas, sino que nos limitamos a presentarlas con la intención de mostrar la heterogeneidad de las propuestas. Son las siguientes:

a) En el EsEnCIAL (bajo el epígrafe 'Indicaciones de uso', p. IX) se utiliza únicamente el término locuciones, sin ofrecer ningún tipo de definición.

b) En el DRAE ('V. Cómo encontrar la definición de expresiones formadas por varios vocablos', p. XXII) se habla de expresiones formadas por varios vocablos y se distingue entre locuciones, frases hechas y modos adverbiales, sin especificar el alcance de cada uno de estos términos. 
c) En el DiPele ('Características generales', p. X), donde se emplea el término genérico fraseología para referirse a este tipo de elementos, se indica que se incluyen en esta categoría las locuciones o construcciones pluriverbales, definidas como "secuencias de palabras en las que no se puede intercambiar el orden de sus componentes"; entre éstas se consideran igualmente las formas compuestas cuyo significado no resulta del de la suma de los elementos que las componen (es el caso de gota fría, ojo de buey...).

d) En el Larousse ('Uso del Gran Diccionario de la Lengua Española', p. XIV) se ofrece una distinción entre locuciones (definidas como "combinaciones estables de dos o más palabras que funcionan como una sola y que pueden equivaler a un adjetivo (loc. adj.), un adverbio (loc. adv.), una conjunción (loc. conj.), o una preposición (loc. prep.)") y frases (explicadas como "proposiciones con significado completo, de forma fija o con flexión en algún elemento, que suelen ser comunes a una comunidad de hablantes"). El repertorio introduce, por otra parte, una tercera clase de elementos, las colocaciones, que no corresponden al concepto que hemos presentado en la introducción, sino a las denominadas formas compuestas o compuestos sintagmáticos, según se desprende de su definición: "secuencias de palabras que suelen ir juntas habitualmente, pero que no tienen una unidad semántica que las caracterice (por ejemplo: agua de colonia, oso panda)".

e) En el ClaVe ('Cómo se usa ClaVE', p. XV) se incluyen, bajo una sola categoría -la de locución - todas aquellas "combinaciones fijas de palabras que forman un solo elemento oracional cuyo significado no es siempre el de la suma de sus miembros" 7 . En este mismo apartado, se indica que, aunque tales locuciones no van acompañadas de indicación gramatical, su definición permite ver si se trata de verbos, sustantivos o adjetivos.

f) En el Salamanca ('Objeto y contenido del diccionario Salamanca', p. VI) se advierte que el usuario encontrará, entre otras informaciones, "numerosas locuciones y frases hechas, los refranes más utilizados en la lengua actual; y muchos ejemplos de uso [...] que muestran muchas colocaciones habituales". Por otra parte, bajo el epígrafe 'Frases y locuciones' (p. XII), se distingue entre a) locuciones de acepción,

7 Al detallar el modo cómo se ordenan las locuciones (ClAVE, p. 2004), se entrevé una distinción entre los conceptos de Locución de acepción y locución de lema que el SALAmANCA, como veremos después, especifica claramente. 
que pueden ser de dos tipos ("las que equivalen semánticamente a la suma de sus miembros" - p. e., guardia urbano, pase de modelos- y "las que no equivalen a sus miembros" -p. e., diente de león, oso hormiguero-) y b) locuciones de lema ("estas frases y locuciones están siempre definidas, llevan la marca del nivel de uso y van acompañadas al menos de un ejemplo"). En ningún momento, por el contrario, se ofrece una definición del concepto de colocación, al que aludíamos más arriba.

3.1.2. Ubicación de las unidades fraseológicas.- La referencia al lugar de aparición de las unidades fraseológicas en el artículo lexicográfico es otro de los elementos habituales en las páginas de presentación y prólogos de los diccionarios estudiados. Sólo en el EsEnCIAL se omite esta información, en parte suplida por la inclusión de una página ('Elementos de las entradas', pp. X-XI) donde se reproducen varios artículos y se especifican los distintos elementos que en él se pueden hallar. También en el DIPELE y en el ClAVE se incorpora una página similar ('Guía para consultar este diccionario'8 y 'Ejemplos de uso de CLAVE' respectivamente), que clarifica e ilustra las precisiones que se realizan en las páginas preliminares.

3.1.3. Abreviaturas y signos lexicográficos.- Las indicaciones o marcas empleadas para caracterizar las unidades fraseológicas se hacen explícitas en un apartado común a todos los diccionarios revisados: la lista de abreviaturas. En cambio, la tipografía y los signos lexicográficos utilizados para distinguir este conjunto de unidades suelen ilustrarse de manera gráfica en la página donde se detallan los diferentes elementos que forman parte del artículo (así puede verse en el EsENCIAL, en el DiPELE y en el CLAVE), aunque también se hacen explícitos por medio de indicaciones de uso, como en el caso del SALAMANCA. Únicamente en el DRAE y en el LAROUSSE se omite cualquier tipo de referencia a las características tipográficas de tales unidades.

3.1.4. Lematización.- Una última cuestión que se aborda habitualmente en las páginas preliminares es la relativa a la lematización de este

${ }^{8}$ En el DiPELE, esta 'Guía' se incluye en las guardas del diccionario, tanto al inicio como al final.

${ }^{9}$ Clave, pp. XVIII-XIX. 
tipo de unidades pluriverbales; sólo en el ESENCIAL y en el LAROUSSE se pasa por alto este elemento de indiscutible necesidad. Acudiendo al criterio de palabra más relevante o palabra gramaticalmente fuerte, en el DRAE, en el DIPELE y en el CLAVE se establece una jerarquía similar, con el siguiente orden: sustantivo (o término sustantivado), verbo, adjetivo, pronombre y adverbio; en el DIPELE no se menciona el pronombre y se incorpora la preposición como último elemento de la serie. Por otra parte, cuando concurren dos o más términos de idéntica categoría en una misma expresión, ésta se incluye, por lo común, bajo el primero de ellos. En el SALAMANCA, por último, se indica que las formas pluriverbales pueden buscarse "por cualquiera de sus palabras clave (sustantivo, adjetivo, verbo), ya que un sistema de remisiones conduce al usuario a la entrada del diccionario en la que están definidas" (p. VII).

\subsection{LA INCLUSIÓN DE LA FRASEOLOGÍA EN EL DICCIONARIO}

El corpus que hemos utilizado para nuestro estudio consta de 30 expresiones, de las cuales 13 son colocaciones ${ }^{10}$ y 17 , locuciones ${ }^{11}$, siguiendo la terminología de Corpas (1997). De lo expuesto en los prólogos se deduce que los diccionarios sólo reconocen explícitamente las segundas (las locuciones), que -como ya hemos apuntado- reúnen los conceptos tradicionales de locución, modismo y frase hecha.

En cuanto a las locuciones, se observa cómo en todos los diccionarios se recoge un número considerable: 17 en el LAROUSSE (la totalidad); 16 en el DraE ${ }^{12}$, el Esencial ${ }^{13}$ y el SALAMANCA ${ }^{14} ; 15$ en el ClaVe ${ }^{15}$;

10 Son las siguientes: (1) dar comienzo, (2) dar un paseo, (3) darse prisa, (4) debate acalorado, (5) decisión clave, (6) declararse un incendio, (7) declararse una epidemia, (8) defender una opinión, (9) defender una postura, (10) desatarse una polémica, (11) desear fervientemente, (12) desempeñar un cargo, (13) dormido profundamente.

11 Son las siguientes: (1) dar calabazas, (2) dar cien vueltas, (3) dar de si, (4) dar gato por liebre, (5) dar y tomar, (6) dar la gana, (7) de/para andar por casa, (8) de armas tomar, (9) de brocha gorda, (10) de golpe y porrazo, (11) de mírame y no me toques, (12) de par en par, (13) de pelo en pecho, (14) de punta en blanco, (15) de rompe y rasga, (16) dorar la pildora, (17) dormir como un tronco.

12 No incluye la (17).

${ }^{13}$ No incluye la (6).

14 No incluye la (2).

15 No incluye la (2) y la (9) 
sólo en el DiPELE 16 se incluyen menos, 13. Estas cifras pueden considerarse ya indicativas del tratamiento lexicográfico dado a las locuciones. Con respecto a las colocaciones, dada la novedad del concepto, los repertorios lexicográficos no dan cuenta explícitamente de este fenómeno léxico y, por lo tanto, no tienen a priori la voluntad de recogerlas. Ahora bien, pueden rastrearse en los ejemplos, en el régimen lexemático que se incluye en la definición o tratadas como locuciones cuando presentan una mayor fijación en la lengua. Teniendo en cuenta estas observaciones, se contabilizan 7 en el SALAMANCA ${ }^{17}, 5$ en el DraE ${ }^{18}$, el LAROUSSE ${ }^{19}$ y el DIPELE $^{20}$, y 3 en el ESENCIAL ${ }^{21}$ y el CLAVE ${ }^{22}$.

\subsection{LAS ESTRUCTURAS DE ACCESO A LA INFORMACIÓN FRASEOLÓGICA}

Para contrastar lo expuesto en los prólogos con la práctica lexicográfica interna, analizaremos, en primer lugar, la palabra ordenatriz de las expresiones; consideraremos después la ubicación y marcación de la fraseología dentro de los artículos; comentaremos, luego, algunas cuestiones relacionadas con la indicación del contorno y, finalmente, nos acercaremos a determinados aspectos formales, así como a las nuevas estructuras de acceso que proporciona el formato electrónico de los diccionarios.

3.3.1. Palabra ordenatriz de las expresiones.- El estudio del corpus pone de manifiesto que aquellos diccionarios que indican en sus páginas preliminares la forma de lematizar (Drae, Dipele, Clave y Salamanca) son en todo momento coherentes con lo allí expuesto. Del análisis se desprende, por otra parte, que en aquellos donde no se mencionan reglas de lematización, esto es, en el ESENCIAL y en el LAROUSSE, se siguen las mismas directrices que en los otros diccionarios, pues se toma también al sustantivo como la categoría gramatical más fuerte. Puede considerarse, por tanto, que la lematización es homogénea en todos los repertorios. Así,

16 No incluye la (1), la (8), la (9) y la (10).

17 Son las siguientes: (2), (3), (5), (8), (9), (1 1) y la (12).

18 Son las siguientes: (2), (3), (6), (7) y (12).

19 Son las siguientes: (1), (2), (3), (7) y (12).

20 Son las siguientes: (2), (3), (5), (6) y (1 2).

21 Son las siguientes: (3), (6) y (7).

22 Son las siguientes: (3), (6) y (12). 
por ejemplo, las expresiones de punta en blanco y de/para andar por casa aparecen sancionadas en los distintos diccionarios bajo los lemas punta y casa, respectivamente.

Es necesario señalar, no obstante, que algunas de las obras lexicográficas examinadas ayudan al usuario en la búsqueda de las expresiones utilizando la técnica de la remisión. Tal es el caso del SALAMANCA, donde se emplea de forma sistemática ${ }^{23}$. Como ya se ha indicado al hablar de los prólogos, en este diccionario las locuciones pueden buscarse por cualquiera de sus palabras claves. Ahora bien, es preciso matizar que las expresiones se encuentran definidas bajo una sola de las entradas. Cuando no se ofrece la definición, van acompañadas de una remisión que conduce al usuario a la entrada bajo la cual se definen ${ }^{24}$. Por ejemplo, la locución dar gato por liebre, se encuentra recogida y definida bajo gato, por ser éste el primero de sus sustantivos, y también se registra en la entrada liebre, acompañada, en este caso, de una remisión al artículo de gato, pues es allí donde se da su significado. Sorprende, sin embargo, el hecho de que algunas expresiones estén definidas bajo más de una entrada. Así ocurre en la locución de armas tomar, la cual se define bajo arma y bajo tomar. En cuanto a las colocaciones, recogidas de forma implícita como antes indicábamos, se encuentran, fundamentalmente, en la entrada del lexema determinante, esto es, del colocativo. Así, por ejemplo, aquellos diccionarios que registran declararse un incendio y declararse una epidemia lo hacen en la entrada de declarar. En ocasiones, también pueden aparecer en el artículo correspondiente al lexema determinado, llamado base. Es el caso de dar un paseo, que en el LAROUSSE se registra bajo el lema paseo. Otra posiblidad es encontrarlas bajo ambos colocados, como ocurre con esta misma expresión en el SAlamanca y en el Dipele.

3.3.2. Ubicación y marcación de la fraseología.- En líneas generales, éste es uno de los aspectos en que se observa un mayor cuidado en los diccionarios de esta década, aunque no siempre se consiga una fór-

23 Por lo que respecta a los restantes diccionarios, hemos podido constatar que tanto en el DRAE como en el ESENCIAL se emplean las remisiones, aunque de forma ocasional.

${ }^{24}$ En este diccionario la remisión se lleva a cabo mediante el empleo de un asterisco $\left({ }^{*}\right)$ sobre la palabra bajo la cual se encuentra definida la expresión. 
mula accesible para el usuario 25 .

Con respecto a la ubicación, los distintos repertorios coinciden en situar las locuciones estudiadas al final del artículo, disponiéndolas alfabéticamente. Cabe precisar, no obstante, que esta ordenación se realiza, bien teniendo en cuenta los espacios en blanco (ESENCIAL, ClaVE, DiPELE y SALAMANCA), bien sin hacerlo (Drae y LaRousSe).

Para marcar la ubicación de la fraseología en el interior del artículo, se utilizan diversas estrategias: en el DRAE y en el DIPELE se sitúa tras las formas compuestas; en el ESENCIAL se subraya con el marbete LOC. y en el SALAMANCA, con FR. Y LOC.; en el LAROUSSE se numera cada una de las expresiones como si se tratara de una acepción más y, por lo tanto, se separa con un salto de línea; en el CLAVE, por último, se incluyen todas bajo una única acepción numerada. En este diccionario, como ya hemos indicado, se ofrece un apéndice ('VI. Ordenación alfabética de las locuciones recogidas en Clave', pp. 2004-2056) en el que, como indica el título, se listan alfabéticamente las locuciones incluidas en el diccionario, teniendo en cuenta todos los elementos de la expresión, así como los espacios en blanco. Junto a la expresión se precisa el lema bajo el que ésta se define. Se trata, a nuestro modo de ver, de un recurso que facilita al usuario la localización de la fraseología, cuando le resulta difícil saber de antemano cuál es el artículo en el que aparece ${ }^{26}$.

Con este mismo objetivo, en los distintos diccionarios se emplean también diversos recursos tipográficos. El principal -y común a todos ellos- es el de grafiar las locuciones en negrilla. Otro de los recursos es la utilización de la doble pleca ( $\|$ ). Este símbolo tipográfico es usado en el CLAVE para indicar, única y exclusivamente, que lo que le sigue es una locución. También se encuentra en el DRAE y en el ESENCIAL; ahora bien, en estos diccionarios, este símbolo no sólo se emplea para indicar la fraseología, sino que cumple otras funciones, como la de separar acepciones (en el DraE) o la de separar categorías (en el EsEnCIAL). Símbolo tipográfico es también el cuadrado negro (ם) utilizado en el DIPELE, que permite identificar con prontitud la fraseología, dado que precede a cada

${ }^{25}$ Es preciso advertir que las consideraciones que realizaremos en este punto se refieren exclusivamente a las denominadas locuciones de lema, puesto que nuestro corpus no comprende locuciones de acepción.

${ }^{26}$ Esto no evita las dificultades que aparecen respecto a las variaciones que pueden tener algunas expresiones, como por ejemplo de armas tomar hay que buscarlo en ser de armas tomar. Sobre esta cuestión volveremos más adelante en el apartado 3.3.7. 
una de las expresiones.

A estas características tipográficas cabe añadir el empleo de abreviaturas. La variedad de soluciones al respecto está en consonancia con lo que ya comentábamos a propósito de los prólogos. Tanto en el DRAE como en el LAROUSSE, las locuciones aparecen acompañadas de la abreviatura "loc.", indicándose a continuación también la categoría gramatical. Es preciso señalar que la mayoría de los diccionarios analizados no contemplan el subtipo de locuciones verbales, sino que aquellas locuciones que equivalen a un verbo son consideradas frases. Por lo que respecta a la marcación de este último tipo de unidades fraseológicas, mientras en el diccionario académico aparecen acompañadas de la abreviatura "fr." (frase), en el LAROUSSE no llevan ninguna. Además de las citadas, en el DRAE también se utiliza, en lugar de fr. y loc., la abreviatura de carácter más general expr. (expresión).

En el ESENCIAL, por otra parte, se emplea la abreviatura "loc." como marbete y se indica la categoría gramatical para cada una de las locuciones, salvo cuando se trata de locuciones verbales. En los restantes diccionarios (ClAVE, DiPELE y SALAMANCA) las locuciones carecen de cualquier tipo de abreviatura, tanto tipológica como categorial. En los diccionarios estudiados las locuciones también se caracterizan, en ocasiones, con otro tipo de marcas no exclusivamente fraseológicas, como por ejemplo las de registro. La consideración de estas cuestiones nos llevaría a tratar otros elementos que excluimos en este trabajo ${ }^{27}$ En cuanto a las colocaciones, cuando se recogen en el ejemplo, figuran en cursiva, y en aquellos casos en que aparecen en la definición, se presentan tipográficamente al igual que el régimen lexemático: en el CLAVE en tipo arial y en el SALAMANCA entre corchetes.

3.3.3. El contorno.- Un aspecto que se relaciona con la forma en que se registran las distintas expresiones y que no se contempla de manera específica en los prólogos es la inclusión o no de lo que Martínez Marín (1991, p. 122) — siguiendo a M. Seco- denomina contorno; esto es, "aquellos elementos que tienen que ver con el contenido combinatorio y contextual de las expresiones". Se trata, entre otros, del sujeto de persona, del sujeto de cosa, y de los complementos de persona y de cosa. En el DRAE, en el ESENCIAL y en el CLAVE se diferencia tipográfica- 
mente el contorno de las expresiones (ej.: "dar cien vueltas a uno"). Así, los elementos estructurales aparecen realzados en negrita redonda, mientras que los del contorno figuran en letra redonda fina. Ahora bien, es necesario señalar que, a veces, podemos encontrarnos con que se incluyen elementos del contorno entre los componentes de la expresión. Así, por ejemplo, en el DRAE la locución dar la gana presenta la forma "darle a uno la gana" en donde el pronombre le ha pasado a ser un componente fijo de la expresión cuando esto realmente no es así, puesto que podemos decir darte la gana, daros la gana, darles la gana, etc. Otro tanto sucede en el EsENCIAL con la expresión dar cien vueltas, pues ésta se registra como "darle a alguien cien vueltas", y no como "dar cien vueltas a alguien", que sería lo adecuado.

Por otra parte, en el diccionario LAROUSSE, si bien se indican los complementos, éstos aparecen en la misma tipografía que los elementos estructurales de las expresiones; por ejemplo, "darle a uno la gana", "dar cien vueltas a alguien".

El SALAMANCA se muestra innovador en este sentido, pues opta por utilizar paréntesis angulares en la definición para indicar el tipo de sujeto que lleva una frase o locución verbal, tal como especifica en la tabla de signos.

Frente a los anteriores diccionarios, en el DIPELE se opta por omitir el contorno de las expresiones. Así, por ejemplo, se registra "dar la gana", sin indicar qué tipo de complementos pueden acompañar a la expresión.

3.3.4. Variantes de las expresiones.- En ocasiones, dentro de una expresión es posible sustituir alguno de sus elementos compositivos sin alterar el significado de la misma. Así, en el DiPELE y en el LAROUSSE se presentan las formas para andar por casa y de andar por casa como variantes de una misma locución. En el primero de los repertorios, la existencia de una variante estructural se indica con una pleca (ej.: "de/para andar por casa") y, en el segundo, a través de la conjunción o (ej.: "de o para andar por casa"). En los restantes diccionarios se ofrece únicamente la forma para andar por casa.

3.3.5. Unidades fraseológicas con casillas vacías.- Se trata de construcciones que contienen una o más casillas libres que pueden ser ocupadas solamente por lexemas pertenecientes a una determinada categoría semántica o gramatical (Varela y Kubarth, 1994, p. XI). Tal es el caso de 
la locución "dar cien vueltas a alguien", donde se advierte una estructura invariable (dar ... vueltas a alguien), y una casilla vacía que puede ser rellenada por palabras como mil, cien, cuarenta mil, etc. En el único diccionario de los analizados que se da cuenta de este fenómeno es en el ESENCIAL, pues se registra la expresión del siguiente modo: "darle a alguien cien vueltas (o cuarenta mil, etc.)".

3.3.6. Elementos opcionales de las expresiones.- Determinados elementos integrantes de las expresiones pueden ser facultativos. De esta manera, es posible decir dar a uno la gana o dar a uno la real gana; de golpe y porrazo o tan sólo de golpe. En algunos de los diccionarios estudiados se informa de este hecho mediante el uso de paréntesis - ClaVE y SALAMANCA-, mientras que en otros se hace a través del empleo de la conjunción disyuntiva o-DrAE y LAROUSSE. Así, por ejemplo, la locución dar a uno la gana, se presenta como "dar la (real) gana" en el SALAMANCA, "dar a alguien la (real) gana" en el ClaVe, "darle a uno la gana o la real gana" en el DRAE y "darle a una persona la gana o la real gana" en el LAROUSSE.

3.3.7. Delimitación de las expresiones.- Ciertas expresiones -fundamentalmente locuciones nominales, adjetivas y adverbiales- pueden presentar restricciones colocacionales con respecto al lexema al cual se aplican; por ejemplo, la locución adverbial de armas tomar se combina frecuentemente con el verbo ser. Ser y de armas tomar forman, pues, una colocación. La mayoría de los diccionarios objeto de estudio no contemplan semejante posibilidad y consideran al verbo ser un elemento estructural de la locución, cuando esto realmente no es así. Otros casos similares que se observan en nuestro corpus son: "hombre de pelo en pecho" 28 (DIPELE) " "pintor de brocha gorda" y "dormir/estar como un tronco" (SALAMANCA), "dormir o quedarse como un tronco" (LAROUSSE), donde nuevamente se incluyen como elementos estructurales las bases de la colocación (hombre, pintor, dormir/estar/quedarse). Esta sería una posible forma de abordar el problema, siempre y cuando los diccionarios especificaran claramente qué elementos forman parte de la expresión y cuáles se colocan con ella.

28 En este diccionario, figura también la expresión "de pelo en pecho" bajo el lema pelo, sin ninguna remisión. 
Otra solución consistiría en ofrecer esta información a través del ejemplo, como de hecho ya puede apreciarse en ciertos casos: (abierto) de par en par (Salamanca), (ponerse) de punta en blanco (Dipele, Clave), (mujer) de rompe y rasga (Salamanca, Dipele, Esencial).

3.3.8. Nuevas estructuras de acceso.- El formato electrónico conque cuentan buena parte de los diccionarios estudiados (DRAE, LAROUSSE y CLAVE) ofrece nuevas posibilidades de acceso a la información fraseológica, que facilitan y agilizan la consulta. En términos generales, las ediciones en CD-ROM de estos repertorios reproducen las indicaciones introducidas en las versiones en papel; sin embargo, todas ellas incorporan un acceso directo a la fraseología, que no permiten las ediciones manuales.

a) En la edición electrónica del DrAE (1995), este acceso se facilita a través del árbol de 'Modos de consulta', donde, entre otras posibilidades, se ofrece una 'Búsqueda de formas complejas'. A través de esta opción se accede a todas las formas pluriverbales del diccionario, independientemente de que se trate de una entrada marcada como loc., fr. $o$ expr. o no esté marcada ${ }^{29}$. Esta es una forma de acceso interesante para el ususario porque introduce, además, entre corchetes, el lema bajo el que se define. Otra opción de acceso es la que facilita el 'Índice de abreviaturas y marcas', que, obviamente, está condicionada por la falta de homogeneidad del repertorio en la asignación y caracterización de las unidades fraseológicas.

b) En el LARousse, bajo el 'Acceso por criterios', se ofrece al usuario la posibilidad de buscar por 'Formas pluriverbales'. Bajo este apartado se distingue entre 'colocaciones' 30 , 'locuciones', 'locuciones de otras lenguas' y 'frases', a partir de la clasificación que se detalla en la edición en papel; y que ahora se facilita en la 'Guía del usuario' de la versión electrónica. Cabe destacar, por otra parte, el uso del color rojo para realzar el tipo escogido, que queda así claramente delimitado del resto.

c) Para finalizar, en el ClaVE se ofrece, entre las opciones de búsqueda, un acceso directo al apartado de 'Locuciones', cuyo contenido

29 Es el caso, por ejemplo, de "hombre de pelo en pecho" que aparece bajo la entrada de hombre sin marca alguna y "de pelo en pecho", marcada como loc. adj., en la entrada de pelo.

${ }^{30}$ Es necesario recordar que el concepto de colocación que utiliza este diccionario no coincide con el nuestro. 
corresponde al listado de cerca de 7000 locuciones que incluye la edición en papel, acompañadas, a diferencia de aquélla, de su correspondiente definición.

\section{CONCLUSIONES}

Las cuestiones que acabamos de analizar nos han permitido observar el tratamiento que algunos de los diccionarios monolingües del español aparecidos en esta década han dado a dos tipos de elementos lexicalizados: locuciones y colocaciones. En primer lugar, hemos podido constatar que falta aún una mayor coherencia en la terminología utilizada, reflejo, en buena parte, de las dificultades teóricas que en este campo se han producido durante bastante tiempo. Esto explica en gran manera la ausencia de sistematicidad en el tratamiento de las colocaciones, un elemento sin duda interesante para los diccionarios de producción, puesto que la competencia de los hablantes nativos $-\mathrm{y}$ por supuesto de los extranjeros-, no siempre llega a suplir la falta de información al respecto. En lo que se refiere a las locuciones estudiadas, puede afirmarse que reciben un tratamiento bastante homogéneo, a pesar de que los prólogos no sean siempre explícitos, en cuanto a su lematización, ubicación y marcación.

La atención particular que hemos prestado a las estructuras de acceso nos ha permitido poner de relieve cuáles son los mecanismos concretos que se ofrecen al usuario para consultar la información sobre fraseología en los diccionarios estudiados. Resulta altamente positivo que se haya conseguido una importante regularización a la hora de determinar cuál es la palabra ordenatriz de las expresiones, aunque esto no siempre vaya acompañado de un sistema coherente de remisiones. La rica variación formal de este tipo de unidades - como hemos puesto de manifiesto en los apartados 3.3.4., 3.3.5., 3.3.6. y 3.3.7.- es una fuente constante de dudas por parte del usuario que debe tener a su alcance recursos para superar este problema; entre éstos cabría señalar la necesidad de aludir de forma clara en las páginas explicativas del diccionario a las decisiones que se han tomado al respecto, de manera que puedan salvarse estos escollos que impiden, en muchas ocasiones, una consulta satisfactoria. En este sentido, las nuevas estructuras de acceso que proporciona el formato electrónico suponen un avance importante, que sin duda ayudarán a subsanar las posibles incoherencias que, en el tratamiento de la fraseología, apreciamos en el texto de partida. 
Los nuevos estudios teóricos han de permitir una consideración más adecuada de los elementos lexicalizados en las obras lexicográficas. Es evidente que, aunque se ha avanzado de manera notable en el tratamiento formal de estos elementos, la falta de coherencia que aún se observa tiene un trasfondo teórico, y éste sólo podrá resolverse atendiendo a los presupuestos que desde la lexicología se están ofreciendo en los últimos años. 


\section{BIBLIOGRAFÍA}

\section{DicCIONARIOS}

Clave: Clave. Diccionario de Uso de Español Actual. Madrid, SM, 1996. (Versión electrónica, 1997).

DiPele: Diccionario para la Enseñanza de la Lengua Española, Barcelona, Vox Biblograf-Universidad de Alcalá de Henares, 1995. DRAE: Real Academia Española, Diccionario de la lengua española, Espasa-Calpe, Madrid, 1992. (Versión electrónica, 1995).

Esencial: Diccionario Esencial Santillana de la Lengua Española, Madrid, Santillana, 1991.

LARousse: Gran Diccionario de la Lengua Española, Barcelona, Larousse Planeta, 1996. (Versión electrónica, 1996).

Salamanca: Diccionario Salamanca de la Lengua Española, Madrid, Santillana-Universidad de Salamanca, 1996.

VARELA, F. y H. KUBARTH (1996): Diccionario fraseológico del español moderno, Madrid, Gredos.

\section{EsTUDIOS}

Bargalló, M. J. Caramés, V. Ferrando y J. M. Moreno (en prensa): "Unidades fraseológicas y diccionarios bilingües", en Actas del III Congreso de Lingüística General (Salamanca, 1997).

CoRpas, G. (1997): Manual de fraseología española, Madrid, Gredos. GARrigA, C. (1997): 'Las 'marcas de uso' en los diccionarios del español”, Revista de investigación lingüística, 1, pp. 75-110.

GutiÉRrez CuADRAdo, J. (1996): "Las marcas en los diccionarios para extranjeros", en P. Díez de Revenga y J. M. Jiménez Cano (eds.), Estudios de Sociolingüística. Sincronía y diacronía, Murcia, DM, pp. 95-106.

MARTíNEZ MARÍN, J. (1991): "Fraseología y diccionarios modernos del español", Voz y Letra, II/1, pp. 117-126.

MARTÍNEZ MARÍN, J. (1996): "Las expresiones fijas en español: perspectivas teoricodescriptiva y aplicada", en Estudios de fraseología española, Málaga, Ágora, pp. 71-85. 